\title{
La interacción humano computador en el currículo de las instituciones de educación superior de Colombia
}

\section{The human computer interaction in the curricula of Colombian higher educative institutions}

\author{
Carlos Andrés Riascos-Pareja ${ }^{1}$ \\ Álvaro Andrés Loaiza-Duque ${ }^{2}$ \\ Royer David Estrada-Esponda ${ }^{3}$
}

Recibido: febrero 12 de 2018

Aceptado: mayo 16 de 2018

\section{Resumen}

El artículo aborda el estado actual de la inclusión de asignaturas referentes a la Interacción Humano Computador, IHC, en los currículos de las Instituciones de Educación superior, IES, de Colombia. La metodología de investigación tuvo un alcance exploratorio y descriptivo, además de tener un corte cuantitativo. La investigación contempló como población objeto de estudio a las 497 IES activas a abril de 2017. Los resultados destacan que al menos 137 IES ofrecen programas del núcleo básico de Ingeniería de Sistemas, Telemáticas y afines, que según el Sistema Nacional de Información de la Educación Superior, SNIES, agrupa a las instituciones que ofrecen programas de formación en el ámbito de la computación. De estas últimas, solo el $28.47 \%$ ofrecen 42 asignaturas relacionadas con la IHC. Además, de la totalidad de egresados en el año 2015 en carreras de dicho grupo, por lo menos el $4.36 \%$ de ellos podrían tener conocimiento de la disciplina. Lo anterior permite concluir que las habilidades o las competencias en el ámbito de la $\mathrm{IHC}$, en los perfiles profesionales de los egresados colombianos, son mínimas en lo que a esta temática se refiere.

Palabras clave: interacción humano computador, $\mathrm{IHC}$, instituciones de educación superior, ingeniería de sistemas, ingeniería de software.

\begin{abstract}
The article addresses the current state of the inclusion of subjects related to the Human Computer Interaction, $\mathrm{HCl}$, in the curricula of Higher Education Institutions, HEl, of Colombia. The research methodology had an exploratory and descriptive scope, besides having a quantitative cut. The research contemplated the 497 active $\mathrm{HEl}$ as a target population to April 2017. The results highlight that at least $137 \mathrm{HEl}$ offer core programs of Computers Engineering, Telematics and related topics, which according to the National Information System of Superior Education, SNIES, group the institutions that offer training programs in the computing field. The latter ones, only $28.47 \%$ offer 42 subjects related to the $\mathrm{HCl}$. In addition, of all the graduates in 2015 from careers of this group, at least $4.36 \%$ of them could have knowledge of the discipline. This allows us to conclude that the skills or competences in the field of the $\mathrm{HCl}$, in the professional profiles of Colombian graduates, are minimal in terms of this topic.
\end{abstract}

Keywords: human computer interaction, $\mathrm{HCl}$, higher education institutions, computers engineering, software engineering.

1 Estudiante de Ingeniería de Sistemas, Universidad del Valle, Tuluá, Colombia. E-mail: carlos.a.riascos@correounivalle.edu.co

2 Estudiante de Ingeniería de Sistemas, Universidad del Valle, Tuluá, Colombia. E-mail: alvaro.andres.loaiza@correounivalle.edu.co

3 Ingeniero de Sistemas, Magíster en Administración, Universidad del Valle, Tuluá, Colombia. E-mail: royer.estrada@correounivalle.edu. co 


\section{Introducción}

Actualmente, en la industria del software se han realizado avances en la definición de modelos, metodologías e implementación de estándares que contribuyen a la evolución y el mejoramiento de la calidad del software (Mercado-Ramos, Zapata \& Ceballos, 2015). Así mismo, autores y organizaciones como: Pressman (2010), Garvin, McCall e ISO25000 (2011), categorizan la calidad del software en atributos claves que se deben tener en cuenta; entre ellos, la usabilidad que contribuye con el mejoramiento de la experiencia de los usuarios mientras usan sistemas informáticos.

En esta industria, las universidades ocupan un papel significativo en la medida en que son ellas las formadoras de profesionales para el campo de la computación. En consecuencia, las Instituciones de Educación Superior, IES, ofrecen programas académicos referentes a la ingeniería de sistemas, ingeniería de software e ingeniería informática; pero en muchos casos, esos programas no incluyen cursos relacionados con: el diseño centrado en el Usuario (DCU), la experiencia de usuario (UX), la experiencia del consumidor (CX), y la usabilidad. Esto a pesar que, por ejemplo, la usabilidad mejora la productividad de los usuarios, reduce los costos de formación y documentación y también permite aumentar la cuota de participación en el mercado (Granollers, Lorés \& Cañas, 2005).

Igualmente, la usabilidad es un factor crítico para la aceptación de un software, debido a que esta permite realizar el trabajo sin esfuerzo, de forma eficiente y evita la frustración de los usuarios. Si estos últimos no perciben el software como una herramienta que ayude a realizar sus tareas con eficiencia, se dificultará la adaptación del sistema, lo que puede generar un uso escaso y olvido del mismo (Pressman, 2010).

En este contexto, importantes organizaciones como, la Asociación Profesional de Especialistas en Información, la User Experience Professionals Association, la Asociación Interacción Perso-
na-Ordenador, la Organización Internacional para la Estandarización y el Special Interest Group on Computer $\neg$ Human Interaction (SIGCHI), se han sumado a la idea de estudiar y divulgar la importancia de la Interacción Humano Computador, $\mathrm{IHC}$, y sus diferentes áreas como la UX, DCU, CX, entre otras, con el fin de aportar técnicas o metodologías que apoyen el desarrollo de software. Esto debido a que la IHC busca mejorar la seguridad, utilidad, efectividad, eficiencia y usabilidad de sistemas que incluyan computadores (Ferreras \& Hayser, 2008).

Respecto a algunos antecedentes significativos, en el ámbito académico se encontró un caso en el que la IHC está incluida en el currículo como un aspecto aislado y no como un aspecto transversal a todo el ciclo de vida del desarrollo de software. En esa experiencia se expone una propuesta sobre un programa de pregrado de ingeniería, en el que se ubica el diseño de interfaces de usuario en el contexto de la ingeniería de software como tópico obligatorio para el plan de estudios (Marco-Simó, Guitart \& Marco-Galindo, 2008).

Asimismo, se encontró una reflexión sobre la puesta en marcha de la propuesta de pregrado presentada por Marco-Simó et al. (2008). Así pues, Marco-Simó, Marco-Galindo y Terrén (2015) señalan que los aspectos relacionados con las interfaces de usuario se abordaron en un curso denominado "Interacción Persona Ordenador", que fue ofrecido en el octavo semestre y obtuvo niveles de rendimiento de más del $74 \%$, entendiendo por rendimiento la razón de estudiantes matriculados versus los estudiantes aprobados; además, los autores comentan que el $70 \%$ de los estudiantes manifestaron estar satisfechos con el curso propuesto. Igualmente, se concluyó que los aspectos de la IHC se deben abordar de manera transversal, porque dicha disciplina no sólo se ubica en el ámbito del desarrollo de software, sino también en el ámbito del diseño y desarrollo de dispositivos, lo cual, de alguna manera, genera dificultades para su integración en los planes curriculares. 
Adicionalmente, se encuentra que en países como España, Brasil y México, se ha otorgado una importancia significativa a la IHC dentro de los currículos académicos, mientras que en Paraguay, Panamá y Perú, apenas es una disciplina emergente (Collazos, Ortega, Granollers, Rusu \& Gutierrez, 2016). Las principales razones identificadas para no incluir la disciplina fueron las siguientes: se requiere habilidad técnica avanzada, no están dentro de los currículos académicos de las universidades, no existe una sinergia entre la academia y la industria, no existe personal cualificado y no hay conocimiento del área. También se observa una incorporación de cursos relacionados con la disciplina, especialmente en los programas de posgrado; sin embargo, a nivel de pregrado, los cursos son complementarios (Granollers, Collazos \& González, 2008).

Igualmente, existen eventos relacionados con el área, tales como: el Congreso Interacción Persona-Ordenador (Interacción), el Encuentro Nacional de Computación, el Interact International Conference y el Latin American Conference on $\mathrm{HCl}$; estos eventos se han realizado en España, Brasil y México, respectivamente. De igual forma, existen grupos o comunidades como AIPO y No Solo Usabilidad, también empresas que ofrecen servicios relacionados con Usabilidad, UX y CX, tales como UserZoom y The Cocktail.

Por otra parte, Collazos y Merchán (2015), realizaron un estudio referente a la incorporación de la IHC en Colombia, tomando como base el ranking de las diez mejores universidades del país. Los autores evidenciaron que solo una de las instituciones ofrece un curso optativo relacionado con la disciplina, lo que lleva a inferir que, a pesar de los esfuerzos por mostrar su importancia, las universidades no están respaldando la integración de la IHC en sus currículos académicos; además, en el estudio no se discriminó si esas universidades ofrecen una carrera incluida dentro del núcleo básico, objeto de interés en la presente investigación.
Igualmente, se encontró que la IHC no es reconocida como un área específica por instituciones como el Instituto Colombiano para el Fomento de la Educación Superior, ICFES. Además, las carreras pueden tener un enfoque en el ámbito de las ciencias de la computación, la ingeniería de software, la ingeniería informática o mixtas, por ende, y gracias a la variedad de mallas curriculares, los comités académicos no la consideran un área de importancia dentro de la computación, sino un capítulo dentro de un curso de ingeniería de software (Villegas, Giraldo, Granollers \& Collazos, 2007). Sin embargo, ACM e IEEE (2017) indican que gracias a la proliferación de servicios web, la computación móvil, las redes sociales y las redes inalámbricas de alta velocidad, una de las innovaciones del marco curricular del reporte Information Technology Curricula 2017, tiene que ver con la User Experience (UX), que está migrando de ámbitos donde las interfaces contienen íconos, ventanas, menús y mouse-clicks, a interfaces hápticas, reconocimiento de gestos y seguimiento de mirada, entre otros.

A pesar de lo anterior, instituciones como la Universidad del Cauca y la Universidad del Quindío, han realizado esfuerzos por incorporar la IHC de manera complementaria en los currículos de los programas de Ingeniería de Sistemas (Villegas, Hernández \& Giraldo, 2009); también han fomentado semilleros de investigación e igualmente han participado en eventos académicos como el Congreso Colombiano de Computación. Esto indica que se ha creado una red de aprendizaje e investigación en torno a la IHC, aunque el ICFES no la reconozca como un área específica de los currículos para la formación de ingenieros del área TI.

Lo anterior justifica la necesidad de abordar la siguiente pregunta de investigación: ¿Cuál es la oferta de cursos relacionados con la IHC, en los currículos de los programas de pregrado, en el área de la computación de las Instituciones de Educación Superior de Colombia? El documento está estructurado de la siguiente manera: primero se presenta la metodología de la investigación, que incluye las fases, el marco muestral, la población 
objeto de estudio y las fuentes de información; luego se presentan los resultados y la discusión; después, se exponen las conclusiones y finalmente se relacionan las referencias bibliográficas que soportaron la investigación.

\section{Metodología}

\subsection{Enfoque y tipo de investigación}

Con el propósito de identificar la cantidad de IES colombianas con programas que ofrecen asignaturas relacionadas con la IHC, User Experience, Customer Experience, Usabilidad y Accesibilidad, y que son importantes para que desde la formación de profesionales en ingeniería de sistemas se aborden temas, prácticas y conocimientos que contribuyan con la calidad de sistemas informáticos, su adecuada implementación en sectores productivos y la comodidad de uso para los usuarios finales, la investigación que aquí se presenta inició con una etapa exploratoria. De acuerdo con Cazau (2006) la investigación exploratoria tiene por objeto abordar problemas de investigación poco estudiados o incluso problemas que nunca han sido objeto de estudio en contextos específicos.

A pesar que han sido identificados estudios de naturaleza similar, en el contexto de esta investigación se aborda específicamente el caso colombiano, y sobre todo se hace énfasis en la totalidad de las IES activas del país. Es decir, no se usaron ránkings $u$ otro tipo de escalafones para desarrollar la investigación. Además, Abreu (2012) menciona que los estudios exploratorios también tienen como propósito el diagnóstico de situaciones, en consecuencia, dicho enfoque metodológico resultó significativo para abordar el cuestionamiento mencionado anteriormente. Además, se utilizó un enfoque cuantitativo, de modo que se desarrollaron actividades relacionadas con la determinación de proporciones y aplicación de cuestionarios; esto con el fin de posibilitar un diagnóstico por medio de la generalización.
Una vez se concluyó la etapa exploratoria y se tuvieron a disposición datos referentes a la inclusión de asignaturas en los currículos de las IES, se dio inicio a una etapa descriptiva que consistió en: la recopilación, tabulación y análisis de los resultados obtenidos, producto de la fase exploratoria; de hecho, Abreu (2012) comenta que la descripción se desencadena de exploraciones creativas. Finalmente, la fase descriptiva posibilitó comprender el estudio cuantitativo y sus implicaciones en los resultados de la investigación.

\subsection{Población y muestra}

La población objeto de estudio estuvo conformada por las 497 IES colombianas activas a abril de 2017, según el Sistema Nacional de la Información de la Educación Superior, SNIES. Por tanto, se usó el tamaño efectivo de la población. El marco muestral estuvo conformado por el listado de las IES colombianas, así como por los listados de programas profesionales ofrecidos por dichas IES.

Además, Azcona, Dorati y Manzini (2013) mencionan que la unidad fundamental de análisis puede ser comprendida "como el tipo de objeto delimitado por el investigador para ser investigado". Por tal motivo, la unidad fundamental de análisis estuvo determinada por las IES colombianas que ofrecen programas profesionales en el ámbito de la computación, específicamente las del núcleo básico Ingeniería de Sistemas, Telemáticas y afines según el SNIES.

\subsection{Recopilación de información}

Se consideraron como fuentes los listados y la información que reposa en el Sistema Nacional de la Información de la Educación Superior, SNIES, y en los portales de las IES colombianas. Estos documentos fueron: listado de IES obtenidas por medio del SNIES, listado de programas profesionales en las IES y sus correspondientes planes de estudio, obtenidos en los portales institucionales. La revisión de dichos portales generó información sobre los planes de estudio asociados a programas 
profesionales de Ingeniería de sistemas, software e informática, objeto de estudio en este proyecto.

Además, por medio de correo electrónico, se envió un cuestionario en línea a los profesores, directivos o encargados de los programas académicos en los que no fue posible identificar las electivas o incluso en los que no fue posible revisar su currículo, con el propósito de fortalecer la recopilación de datos. En la tabla 1 se presentan las preguntas más significativas para el análisis, así como el tipo de variable y su respectivo objetivo.

\begin{tabular}{|c|c|c|}
\hline Pregunta & Tipo & Objetivo \\
\hline $\begin{array}{l}\text { En su Universidad se ofrecen CURSOS COMPLETOS } \\
\text { relacionados a disciplinas como: Interacción Per- } \\
\text { sona-Ordenador, Diseño de Interfaces de Usuario, } \\
\text { Diseño Centrado en el Usuario, Usabilidad o Acce- } \\
\text { sibilidad. }\end{array}$ & Dicotómica & $\begin{array}{l}\text { Determinar la proporción de } \\
\text { IES activas en Colombia que } \\
\text { ofrecen programas de esa na- } \\
\text { turaleza }\end{array}$ \\
\hline $\begin{array}{l}\text { ¿Por qué no se ofrece un curso relacionado a la } \\
\text { disciplina de Interacción Persona-Ordenador? }\end{array}$ & No estructurada & $\begin{array}{l}\text { Determinar las motivaciones } \\
\text { del porqué no se ofrecen asig- } \\
\text { naturas relacionadas con la IHC } \\
\text { como disciplina }\end{array}$ \\
\hline $\begin{array}{l}\text { ¿Tienen iniciativas o proyectos a futuro de ofertar } \\
\text { cursos relacionados a la INTERACCIÓN PERSONA- } \\
\text { ORDENADOR en su Universidad? }\end{array}$ & $\begin{array}{l}\text { Dicotómica - No } \\
\text { estructurada }\end{array}$ & $\begin{array}{l}\text { Conocer las posibles motiva- } \\
\text { ciones de las IES para ofrecer } \\
\text { o no cursos de esta naturaleza. }\end{array}$ \\
\hline
\end{tabular}

Tabla 1. Preguntas realizadas en la fase de consulta.

Con el fin de cotejar las respuestas enviadas por los directivos, se procedió a filtrar y chequear cada una de las respuestas recibidas, con la intención de suprimir aquellas que se consideraron repetidas o no válidas. En la fase de análisis fue importante la clasificación propuesta en Granollers (2004), porque menciona que alrededor de la IHC se encuentran áreas de conocimiento o disciplinas, que se relacionan directamente con esta y tienen aportes que son necesarios para desarrollar sistemas interactivos usables y accesibles, en los que las personas y sus tareas son los principales objetivos. Estas áreas son: Etnografía, Sociología, Psicología, Diseño gráfico, Programación, Ingeniería de software, Inteligencia artificial, Documentación y Ergonomía.

\section{Resultados y discusión}

El SNIES clasifica a las IES en: Institución Técnica Profesional, Institución Tecnológica, Institución Universitaria/Escuela Tecnológica y Universidades. Además, en la consulta realizada se identificó que una Institución Técnica Profesional, así como una Institución Tecnológica, ofrecen carreras profesionales. En consecuencia, la población de IES consideradas las incluyó a todas sin distinguir su categoría.

Con base en lo anterior, el número total de IES activas a abril de 2017 corresponde a 497; de estas, 137 ofrecen programas relacionados con en el núcleo básico de Ingeniería de Sistemas y Telemáticas. La figura 1 da cuenta de las IES colombianas activas e incluye la cantidad de ellas que ofrecen programas activos, como: Ingeniería de sistemas, Ingeniería en informática, Ingeniería de software y, en general, los incluidos en el núcleo básico de Ingeniería de Sistemas, Telemáticas y afines, según la clasificación del SNIES. 


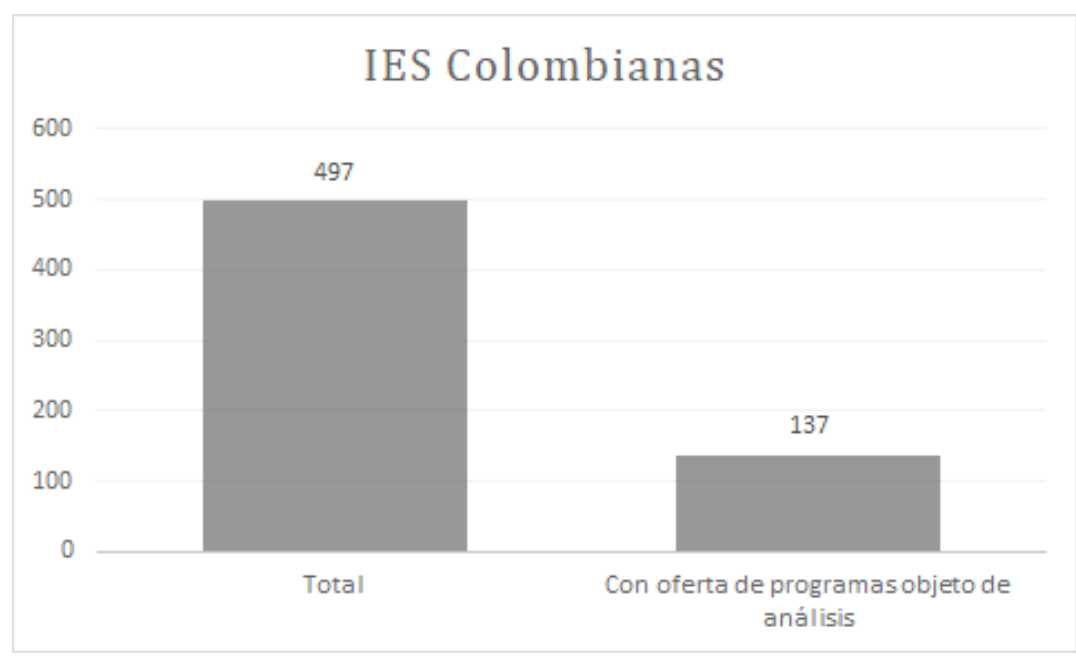

Figura 1. Número de instituciones con oferta de programas del núcleo básico de ing. de sistemas y afines.

Una vez revisados los portales institucionales de las IES, se encontró que de las 137 solo 22 ofrecen 26 cursos relacionados con la IHC; 20 de ellas no ofrecen cursos de esa naturaleza y en las 95 restantes no fue posible identificar si ofrecen o no cursos relacionados.

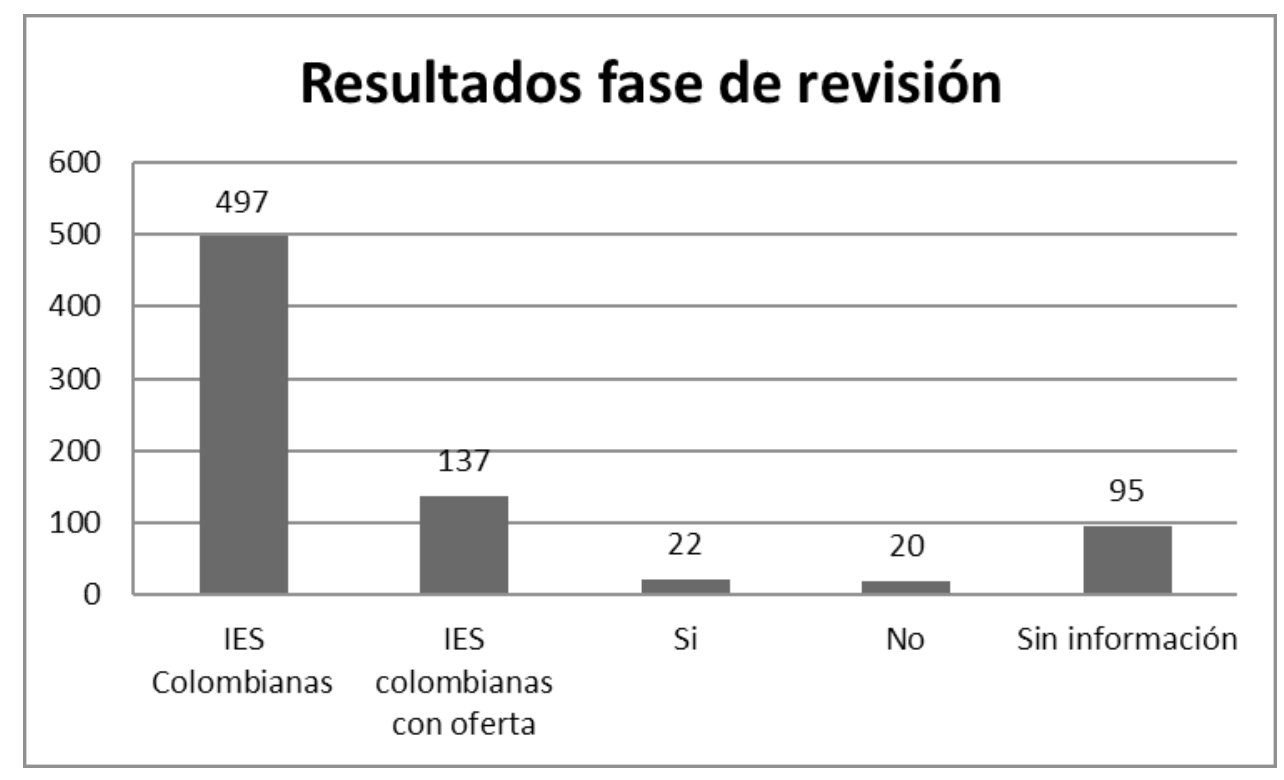

Figura 2. Resultados de revisión de portales web institucionales.

La figura 2 resume los datos obtenidos después de la etapa de revisión, en cuanto al número de IES que ofrecen asignaturas relacionadas con la IHC. Adicionalmente, la tabla 2 presenta a cada una de ellas y además da cuenta, en la mayoría de los casos, del tipo de asignatura, el semestre en el que se ofrece y la cantidad de créditos académicos asignados. Entendiendo que la IHC es de naturaleza interdisciplinar (Granollers, 2004), se decidió clasificar las asignaturas según su área de interés para facilitar el análisis y proveer resultados a la luz de dicha naturaleza. 


\begin{tabular}{|c|c|c|c|c|c|c|}
\hline Universidad & $\begin{array}{l}\text { Programa } \\
\text { académico }\end{array}$ & Asignatura & Tipo & Semestre & Créditos & Categoría \\
\hline $\begin{array}{l}\text { Universidad de la } \\
\text { Sabana }\end{array}$ & $\begin{array}{l}\text { Ingeniería Infor- } \\
\text { mática }\end{array}$ & $\begin{array}{l}\text { Interacción Huma- } \\
\text { no Computador }\end{array}$ & Obligatoria & VI & 3 & $\mathrm{IHC}$ \\
\hline $\begin{array}{l}\text { Universidad Industrial } \\
\text { de }\end{array}$ & $\begin{array}{l}\text { Ingeniería de } \\
\text { Sistemas }\end{array}$ & $\begin{array}{l}\text { Interacción Hom- } \\
\text { bre Computador }\end{array}$ & Electiva & $\mathrm{S} / \mathrm{N}$ & 4 & $\mathrm{IHC}$ \\
\hline \multicolumn{7}{|l|}{ Santander } \\
\hline $\begin{array}{l}\text { Corporación Tecnoló- } \\
\text { gica Industrial Colom- } \\
\text { biana - TEINCO }\end{array}$ & $\begin{array}{l}\text { Ingeniería de } \\
\text { Sistemas }\end{array}$ & $\begin{array}{l}\text { Herramientas gráfi- } \\
\text { cas de software }\end{array}$ & Obligatoria & II & $\mathrm{S} / \mathrm{N}$ & Diseño gráfico \\
\hline $\begin{array}{l}\text { Corporación Universi- } \\
\text { taria Lasallista }\end{array}$ & $\begin{array}{l}\text { Ingeniería Infor- } \\
\text { mática }\end{array}$ & $\begin{array}{l}\text { Diseño y edición } \\
\text { gráfica }\end{array}$ & Obligatoria & II & 2 & Diseño gráfico \\
\hline $\begin{array}{l}\text { Corporación Universi- } \\
\text { taria Minuto de Dios } \\
\text { - UNIMINUTO }\end{array}$ & $\begin{array}{l}\text { Ingeniería de } \\
\text { Sistemas }\end{array}$ & Diseño gráfico & Obligatoria & IV & 3 & Diseño gráfico \\
\hline $\begin{array}{l}\text { Corporación Universi- } \\
\text { taria Reformada - CUR }\end{array}$ & $\begin{array}{l}\text { Ingeniería Infor- } \\
\text { mática }\end{array}$ & $\begin{array}{l}\text { Desarrollo de inter- } \\
\text { faces gráficas }\end{array}$ & Obligatoria & IV & 3 & $\begin{array}{l}\text { Diseño de } \\
\text { interfaces de } \\
\text { usuario }\end{array}$ \\
\hline $\begin{array}{l}\text { Fundación para la } \\
\text { educación superior } \\
\text { San Mateo }\end{array}$ & $\begin{array}{l}\text { Ingeniería de } \\
\text { Sistemas }\end{array}$ & $\begin{array}{l}\text { Desarrollo de inter- } \\
\text { faces gráficas }\end{array}$ & $\mathrm{S} / \mathrm{N}$ & $\mathrm{S} / \mathrm{N}$ & $\mathrm{S} / \mathrm{N}$ & $\begin{array}{l}\text { Diseño de } \\
\text { interfaces de } \\
\text { usuario }\end{array}$ \\
\hline $\begin{array}{l}\text { Fundación Universita- } \\
\text { ria Konrad Lorenz }\end{array}$ & $\begin{array}{l}\text { Ingeniería de } \\
\text { Sistemas }\end{array}$ & $\begin{array}{l}\text { Diseño de Interfa- } \\
\text { ces de Usuario }\end{array}$ & Obligatoria & IV & $\mathrm{S} / \mathrm{N}$ & $\begin{array}{l}\text { Diseño de } \\
\text { interfaces de } \\
\text { usuario }\end{array}$ \\
\hline $\begin{array}{l}\text { Universidad Coopera- } \\
\text { tiva de Colombia }\end{array}$ & $\begin{array}{l}\text { Ingeniería de } \\
\text { Sistemas }\end{array}$ & $\begin{array}{l}\text { Diseño de Interfa- } \\
\text { ces de Software }\end{array}$ & Obligatoria & V & 3 & $\begin{array}{l}\text { Diseño de } \\
\text { interfaces de } \\
\text { usuario }\end{array}$ \\
\hline $\begin{array}{l}\text { Corporación Univer- } \\
\text { sitaria Autónoma de } \\
\text { Nariño -AUNAR }\end{array}$ & $\begin{array}{l}\text { Ingeniería Infor- } \\
\text { mática }\end{array}$ & $\begin{array}{l}\text { Técnicas del diseño } \\
\text { hipermedia en la } \\
\text { informática }\end{array}$ & Obligatoria & V & 4 & Diseño gráfico \\
\hline \multirow{2}{*}{$\begin{array}{l}\text { Universidad Manuela } \\
\text { Beltrán }\end{array}$} & \multirow{2}{*}{$\begin{array}{l}\text { Ingeniería de } \\
\text { Software }\end{array}$} & $\begin{array}{l}\text { Diseño de Inter- } \\
\text { faces }\end{array}$ & Obligatoria & IX & $\mathrm{S} / \mathrm{N}$ & $\begin{array}{l}\text { Diseño de } \\
\text { interfaces de } \\
\text { usuario }\end{array}$ \\
\hline & & $\begin{array}{l}\text { Arquitecturas de } \\
\text { información y } \\
\text { Usabilidad }\end{array}$ & Obligatoria & $x$ & 3 & $\mathrm{IHC}$ \\
\hline $\begin{array}{l}\text { Universidad Santo } \\
\text { Tomás }\end{array}$ & $\begin{array}{l}\text { Ingeniería en Infor- } \\
\text { mática }\end{array}$ & $\begin{array}{l}\text { Usabilidad y } \\
\text { accesibilidad en } \\
\text { entornos tecnoló- } \\
\text { gicos }\end{array}$ & Obligatoria & IIX & 4 & $\mathrm{IHC}$ \\
\hline \multirow{2}{*}{$\begin{array}{l}\text { Pontificia Universidad } \\
\text { Javeriana }\end{array}$} & \multirow{2}{*}{$\begin{array}{l}\text { Ingeniería de } \\
\text { Sistemas }\end{array}$} & $\begin{array}{l}\text { Interacción Hom- } \\
\text { bre Máquina - }\end{array}$ & Obligatoria & IV & 3 & $\mathrm{IHC}$ \\
\hline & & $\begin{array}{l}\text { Diseño de inter- } \\
\text { faces Humano } \\
\text { Computador }\end{array}$ & $\mathrm{S} / \mathrm{N}$ & $\mathrm{S} / \mathrm{N}$ & $\mathrm{S} / \mathrm{N}$ & $\begin{array}{l}\text { Diseño de } \\
\text { interfaces de } \\
\text { usuario }\end{array}$ \\
\hline
\end{tabular}




\begin{tabular}{|c|c|c|c|c|c|c|}
\hline Universidad & $\begin{array}{l}\text { Programa } \\
\text { académico }\end{array}$ & Asignatura & Tipo & Semestre & Créditos & Categoría \\
\hline $\begin{array}{l}\text { Universidad Autóno- } \\
\text { ma de } \\
\text { Bucaramanga }\end{array}$ & $\begin{array}{l}\text { Gestión de Siste- } \\
\text { mas de Informa- } \\
\text { ción }\end{array}$ & $\begin{array}{l}\text { Interfaces e Inte- } \\
\text { racción Hombre } \\
\text { Máquina }\end{array}$ & Obligatoria & VII & 2 & $\mathrm{IHC}$ \\
\hline \multirow{2}{*}{$\begin{array}{l}\text { Universidad Autóno- } \\
\text { ma de Occidente }\end{array}$} & \multirow{2}{*}{$\begin{array}{l}\text { Ingeniería Multi- } \\
\text { media }\end{array}$} & $\begin{array}{l}\text { Interacción Hom- } \\
\text { bre Máquina }\end{array}$ & Obligatoria & VII & 4 & $\mathrm{IHC}$ \\
\hline & & $\begin{array}{l}\text { Ingeniería de la } \\
\text { Usabilidad }\end{array}$ & Electiva & $\mathrm{S} / \mathrm{N}$ & $\mathrm{S} / \mathrm{N}$ & $\mathrm{IHC}$ \\
\hline Universidad de Caldas & $\begin{array}{l}\text { Ingeniería de } \\
\text { Sistemas y Compu- } \\
\text { tación }\end{array}$ & $\begin{array}{l}\text { Diseño de Inter- } \\
\text { faces }\end{array}$ & Obligatoria & $\mathrm{S} / \mathrm{N}$ & 2 & $\begin{array}{l}\text { Diseño de } \\
\text { interfaces de } \\
\text { usuario }\end{array}$ \\
\hline $\begin{array}{l}\text { Universidad de Carta- } \\
\text { gena }\end{array}$ & $\begin{array}{l}\text { Ingeniería de } \\
\text { Software }\end{array}$ & $\begin{array}{l}\text { Interacción Huma- } \\
\text { no Computador }\end{array}$ & Obligatoria & VIII & 3 & $\mathrm{IHC}$ \\
\hline $\begin{array}{l}\text { Universidad de San- } \\
\text { tander }\end{array}$ & $\begin{array}{l}\text { Ingeniería de } \\
\text { Software }\end{array}$ & $\begin{array}{l}\text { Interacción Hom- } \\
\text { bre - Computador }\end{array}$ & Obligatoria & IX & $\mathrm{S} / \mathrm{N}$ & $\mathrm{IHC}$ \\
\hline \multirow{2}{*}{ Universidad Del Valle } & \multirow{2}{*}{$\begin{array}{l}\text { Ingeniería de } \\
\text { Sistemas }\end{array}$} & $\begin{array}{l}\text { Usabilidad de } \\
\text { Interfaces Humano } \\
\text { Computador }\end{array}$ & Electiva & VIII & 3 & $\mathrm{IHC}$ \\
\hline & & $\begin{array}{l}\text { Diseño de Interfa- } \\
\text { ces de Usuario }\end{array}$ & Electiva & V & 3 & $\begin{array}{l}\text { Diseño de } \\
\text { interfaces de } \\
\text { usuario }\end{array}$ \\
\hline $\begin{array}{l}\text { Universidad Distrital } \\
\text { Francisco José de } \\
\text { Caldas }\end{array}$ & $\begin{array}{l}\text { Ingeniería de } \\
\text { Sistemas }\end{array}$ & Factores Humanos & Electiva & IX & 3 & $\mathrm{IHC}$ \\
\hline $\begin{array}{l}\text { Instituto Universitario } \\
\text { de la Paz }\end{array}$ & $\begin{array}{l}\text { Ingeniería Infor- } \\
\text { mática }\end{array}$ & Diseño gráfico & $\mathrm{S} / \mathrm{N}$ & $\mathrm{S} / \mathrm{N}$ & $\mathrm{S} / \mathrm{N}$ & Diseño gráfico \\
\hline Universidad Del Norte & $\begin{array}{l}\text { Ingeniería de } \\
\text { Sistemas y Compu- } \\
\text { tación }\end{array}$ & Diseño Digital & $\mathrm{S} / \mathrm{N}$ & $\mathrm{S} / \mathrm{N}$ & $\mathrm{S} / \mathrm{N}$ & Diseño gráfico \\
\hline
\end{tabular}

Tabla 2. Cursos ofertados en las IES, relacionados con IHC.

Gracias a la revisión fue posible identificar que 22 IES, es decir, el $16.05 \%$ del total de instituciones que ofrecen programas objeto de estudio, cuen$\tan$ con un total de 26 asignaturas relacionadas con la IHC o con áreas de conocimiento comple- mentarias a la misma. Adicionalmente, y según la denominación de los cursos, fue posible categorizar dichas asignaturas, como se muestra en la figura 3. 


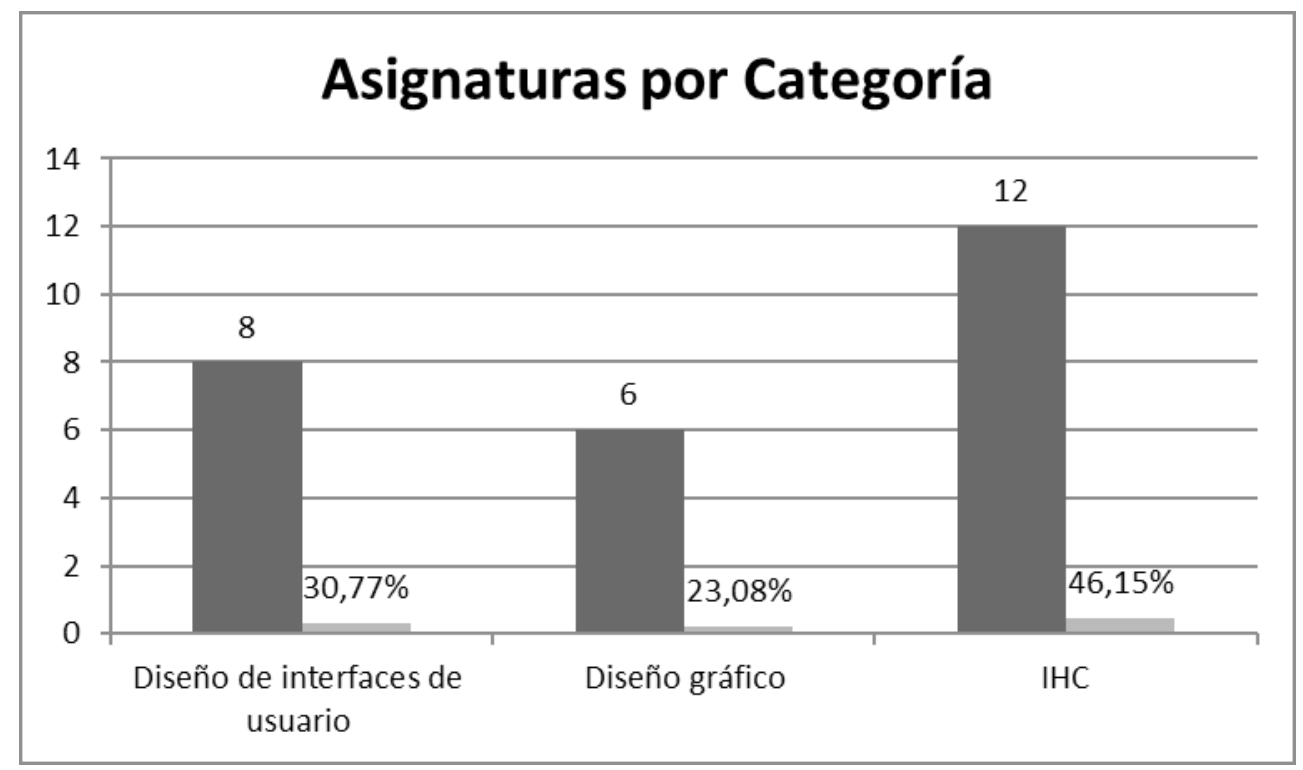

Figura 3. Asignaturas por categoría - fase de revisión.

La categoría denominada Diseño de Interfaces de usuario, agrupa cursos que según su nombre dan cuenta de planes de estudio que conducen a dotar a los estudiantes de habilidades, técnicas y herramientas que buscan desarrollar interfaces gráficas para los sistemas informáticos que producen, pero no necesariamente implica que en tales cursos se aborde la IHC de manera integral. Es decir, la denominación de tales cursos no implica el abordaje de disciplinas como etnografía, sociología, ergonomía, inteligencia artificial, entre otras, que son fundamentales para diseñar sistemas interactivos usables y accesibles.

Por otra parte, la categoría Diseño Gráfico agrupa a las asignaturas que, según su denominación, se puede inferir que apelan por enfoques más instrumentales. La categoría IHC agrupa los cursos cuya denominación guarda mayor fidelidad con la disciplina de manera integral, esto permite inferir que son planeados y ejecutados de una manera holística en relación con dicha disciplina, considerando que el diseño de sistemas interactivos requiere de grupos y habilidades de naturaleza interdisciplinar.

Teniendo en cuenta estos resultados, es prudente comentar que la mayoría de los portales institucionales de las IES no ofrece suficiente información sobre sus pénsum académicos; esto conduce a considerar que las instituciones deberían publicar tal información, con el fin de facilitar el proceso de revisión que los futuros estudiantes seguramente realizarán a los programas académicos que pretendan iniciar. La figura 4 sintetiza los resultados obtenidos de la fase de consulta, en la que se envió un cuestionario digital, con el fin de indagar sobre el tema objeto de estudio a las IES en las que no fue posible obtener información por medio de sus portales institucionales. 


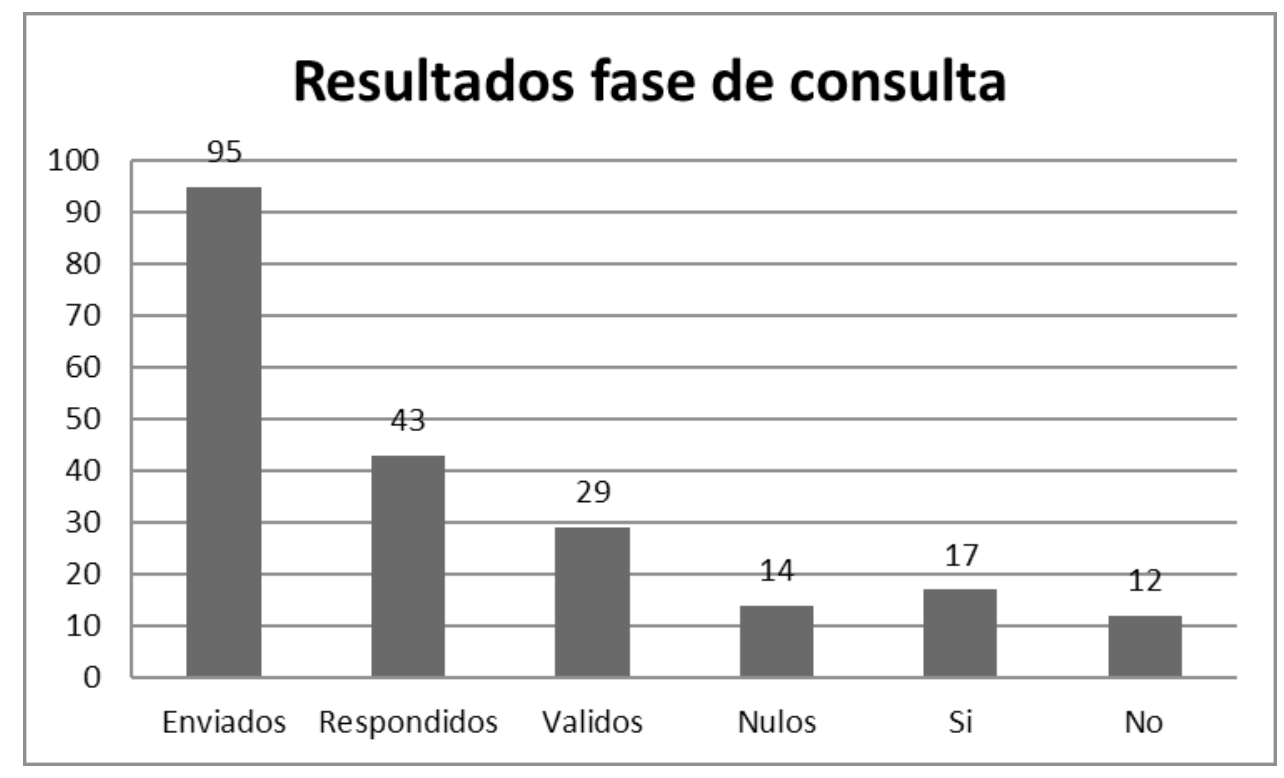

Figura 4. Resultados fase de consulta.

Es importante mencionar que las 14 respuestas nulas de la figura 4, corresponden a comentarios provenientes de estudiantes; dichas respuestas evidencian que los directivos, profesores o encargados de los programas académicos que recibieron el correo trasladaron el cuestionario a sus estudiantes. A pesar de que los comentarios de los estudiantes son significativos y de hecho sus respuestas en su mayoría muestran expectativas positivas en relación con la IHC, el cuestionario estaba dirigido a personas de los estamentos administrativo o profesoral, quienes finalmente son los que toman decisiones en asuntos de naturaleza curricular. Lo anterior conduce a pensar que para el caso del cuestionario es posible mejorar su consigna principal, con el fin de evitar este tipo de eventos en el caso de la reproducción de este estudio. Adicionalmente, la tabla 3 relaciona a todas las IES que manifestaron ofrecer en sus programas académicos asignaturas afines con la IHC.

\begin{tabular}{|c|c|c|c|c|c|c|}
\hline Universidad & $\begin{array}{c}\text { Programa acadé- } \\
\text { mico }\end{array}$ & Asignatura & Tipo & Semestre & Créditos & Categoría \\
\hline $\begin{array}{l}\text { Corporación Universi- } \\
\text { taria Rafael Núñez }\end{array}$ & $\begin{array}{l}\text { Ingeniería de } \\
\text { Sistemas }\end{array}$ & Diseño de Software & Obligatoria & V & 3 & $\begin{array}{l}\text { Ingeniería de } \\
\text { Software }\end{array}$ \\
\hline $\begin{array}{l}\text { Universidad de } \\
\text { Boyacá }\end{array}$ & $\begin{array}{l}\text { Ingeniería de } \\
\text { Sistemas }\end{array}$ & $\begin{array}{l}\text { Ingeniería del sof- } \\
\text { tware I }\end{array}$ & Obligatoria & V & 3 & $\begin{array}{l}\text { Ingeniería de } \\
\text { Software }\end{array}$ \\
\hline $\begin{array}{l}\text { Universidad Militar } \\
\text { Nueva Granada }\end{array}$ & $\begin{array}{l}\text { Ingeniería en } \\
\text { Multimedia }\end{array}$ & $\begin{array}{l}\text { Movimiento e inte- } \\
\text { racción }\end{array}$ & Electiva & IX & 3 & Ergonomía \\
\hline $\begin{array}{l}\text { Fundación Universita- } \\
\text { ria María Cano }\end{array}$ & $\begin{array}{l}\text { Ingeniería de } \\
\text { Software }\end{array}$ & $\begin{array}{l}\text { Ingeniería del sof- } \\
\text { tware }\end{array}$ & Obligatoria & V & 3 & $\begin{array}{l}\text { Ingeniería de } \\
\text { Software }\end{array}$ \\
\hline Universidad Mariana & $\begin{array}{l}\text { Ingeniería de } \\
\text { Sistemas }\end{array}$ & $\begin{array}{l}\text { Interacción Hombre } \\
\text { Máquina }\end{array}$ & Obligatoria & IV & 3 & $\mathrm{IHC}$ \\
\hline $\begin{array}{l}\text { Universidad Autóno- } \\
\text { ma de Occidente }\end{array}$ & $\begin{array}{l}\text { Ingeniería Infor- } \\
\text { mática }\end{array}$ & $\begin{array}{l}\text { Ingeniería de la } \\
\text { Usabilidad }\end{array}$ & Electiva & $\begin{array}{l}\text { Cumpli- } \\
\text { dos } 100 \\
\text { créditos }\end{array}$ & 3 & Usabilidad \\
\hline $\begin{array}{l}\text { Universidad Antonio } \\
\text { Nariño }\end{array}$ & $\begin{array}{l}\text { Ingeniería de } \\
\text { Sistemas }\end{array}$ & Inteligencia Artificial & Obligatoria & VII & 4 & $\begin{array}{l}\text { Inteligencia } \\
\text { Artificial }\end{array}$ \\
\hline
\end{tabular}




\begin{tabular}{|c|c|c|c|c|c|c|}
\hline Universidad & $\begin{array}{c}\text { Programa acadé- } \\
\text { mico }\end{array}$ & Asignatura & Tipo & Semestre & Créditos & Categoría \\
\hline $\begin{array}{l}\text { Universidad Popular } \\
\text { del Cesar }\end{array}$ & $\begin{array}{l}\text { Ingeniería de } \\
\text { sistemas }\end{array}$ & $\begin{array}{l}\text { Ingeniería de sof- } \\
\text { tware }\end{array}$ & Obligatoria & V & 4 & $\begin{array}{l}\text { Ingeniería de } \\
\text { Software }\end{array}$ \\
\hline $\begin{array}{l}\text { Universidad Francisco } \\
\text { de Paula Santander }\end{array}$ & $\begin{array}{l}\text { Ingeniería de } \\
\text { Sistemas }\end{array}$ & $\begin{array}{l}\text { Análisis y Diseño de } \\
\text { Sistemas }\end{array}$ & Obligatoria & VII & 4 & $\begin{array}{l}\text { Ingeniería de } \\
\text { Software }\end{array}$ \\
\hline $\begin{array}{l}\text { Universidad Tecnoló- } \\
\text { gica de Bolívar }\end{array}$ & $\begin{array}{l}\text { Ingeniería de } \\
\text { Sistemas }\end{array}$ & $\begin{array}{l}\text { Diseño de Ambien- } \\
\text { tes Web }\end{array}$ & Electiva & VI & 3 & $\begin{array}{l}\text { Ingeniería de } \\
\text { Software }\end{array}$ \\
\hline $\begin{array}{l}\text { Instituto Tolimense } \\
\text { de Formación técnica } \\
\text { profesional. ITFIP }\end{array}$ & $\begin{array}{l}\text { Ingeniería de } \\
\text { Sistemas }\end{array}$ & $\begin{array}{l}\text { Lógica de progra- } \\
\text { mación }\end{array}$ & Obligatoria & I & 3 & Programación \\
\hline $\begin{array}{l}\text { Universidad de } \\
\text { Bogotá Jorge Tadeo } \\
\text { Lozano }\end{array}$ & $\begin{array}{l}\text { Ingeniería de } \\
\text { Sistemas }\end{array}$ & $\begin{array}{l}\text { Gerencia de Pro- } \\
\text { yectos }\end{array}$ & Obligatoria & VIII & 3 & Proyectos \\
\hline $\begin{array}{l}\text { Universidad de San } \\
\text { Gil }\end{array}$ & $\begin{array}{l}\text { Ingeniería de } \\
\text { sistemas }\end{array}$ & $\begin{array}{l}\text { Ingeniería de Sof- } \\
\text { tware }\end{array}$ & Obligatoria & VII & 4 & $\begin{array}{l}\text { Ingeniería de } \\
\text { Software }\end{array}$ \\
\hline $\begin{array}{l}\text { Corporación Uni- } \\
\text { versitaria del Caribe. } \\
\text { CECAR }\end{array}$ & $\begin{array}{l}\text { Ingeniería de } \\
\text { sistemas }\end{array}$ & Análisis y Diseño II & Obligatoria & VII & 3 & $\begin{array}{l}\text { Ingeniería de } \\
\text { Software }\end{array}$ \\
\hline $\begin{array}{l}\text { Universidad Coopera- } \\
\text { tiva de Colombia }\end{array}$ & $\begin{array}{l}\text { Ingeniería de } \\
\text { sistemas }\end{array}$ & Diseño de interfaces & Obligatoria & IX & 2 & $\begin{array}{l}\text { Diseño de inter- } \\
\text { faces de usuarios }\end{array}$ \\
\hline $\begin{array}{l}\text { Universidad Católica } \\
\text { de Colombia }\end{array}$ & $\begin{array}{l}\text { Ingeniería de Sis- } \\
\text { temas y Compu- } \\
\text { tación }\end{array}$ & $\begin{array}{l}\text { Interacción Huma- } \\
\text { no-Computador }\end{array}$ & Electiva & IIX - IX - X & 3 & $\mathrm{IHC}$ \\
\hline $\begin{array}{l}\text { Universidad Coopera- } \\
\text { tiva de Colombia }\end{array}$ & $\begin{array}{l}\text { Ingeniería de } \\
\text { Sistemas }\end{array}$ & $\begin{array}{l}\text { Desarrollo de dispo- } \\
\text { sitivos móviles }\end{array}$ & Electiva & $I I X-I X-X$ & 3 & $\begin{array}{l}\text { Ingeniería de } \\
\text { Software }\end{array}$ \\
\hline
\end{tabular}

Tabla 3. Cursos ofertados por las IES - fase consulta.

En la fase de consulta fue posible agrupar las 17 asignaturas en 6 categorías adicionales. En primera instancia, se advierte que una de las categorías, en este caso la denominada Proyectos, sale del contexto de la IHC, o por lo menos eso se infiere, ya que un curso de gestión de proyectos se en- foca en gestionar las personas, los productos, los procesos y el proyecto mismo con el fin de lograr el éxito (Pressman, 2010). La figura 5 presenta la totalidad de asignaturas catalogadas en la fase de consulta. 


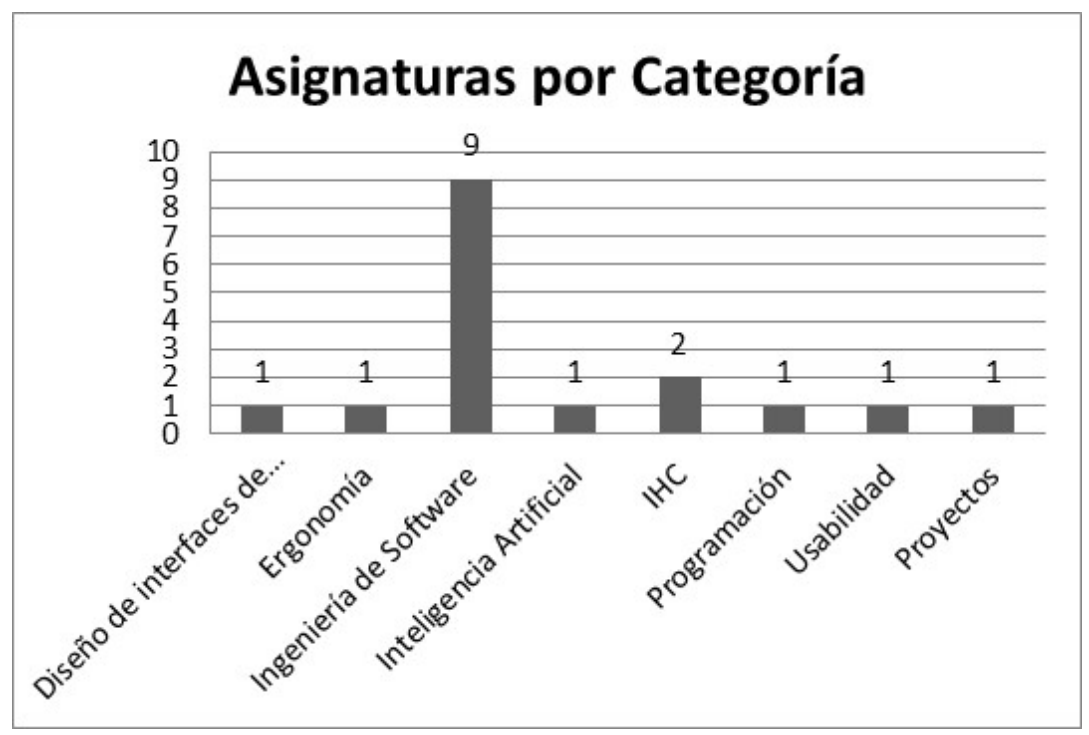

Figura 5. Asignaturas por categoría - fase consulta.

Todas las categorías -exceptuando Proyectos- guardan relación directa con la IHC; este es el caso de la Ergonomía, Ingeniería de software, Inteligencia artificial, Programación y Usabilidad; de hecho, tales áreas de conocimiento son vistas como componentes significativos para el desarrollo de sistemas interactivos (Granollers, 2004). Por otra parte, se destaca que el $52.94 \%$ de las asignaturas tuvieron lugar en la categoría Ingeniería de Software, lo cual conduce a pensar que es posible que el diseño de sistemas interactivos, desde la perspectiva de la IHC, es considerado aún como un apéndice dentro del proceso ingenieril de desarrollo software y no de manera holística; por tanto, no se apela por enfoques o metodologías que combinen ambas disciplinas. Solo en dos respuestas, las de la Universidad Católica de Manizales y la Universidad Mariana, es coherente pensar que se considera a la IHC de manera integral en el desarrollo de sistemas interactivos.

Al igual que en la fase de Revisión, se observó cierto grado de confidencialidad en relación con la publicación de los pénsum académicos por parte de las IES; incluso se recibieron respuestas en las que explícitamente se manifestó que tal información era de dominio privado o simplemente era necesario surtir un mayor número de procedimientos administrativos para recibirla. Lo anterior conduce a re- flexionar desde dos perspectivas: la primera relacionada con una clara y respetable reserva sobre los planes académicos o la segunda y menos probable, el inadecuado uso del cuestionario diseñado para indagar sobre la oferta de asignaturas relacionadas con la IHC.

Teniendo en cuenta los resultados de las fases de Revisión y Consulta, se destaca que, de 137 IES, sólo en 39 de ellas se ofrecen asignaturas con una visión integral de la IHC, esto según la denominación de sus cursos, o como áreas de conocimiento complementarias a tal disciplina; es decir, sólo el $28.47 \%$ de los currículos incluyen asignaturas de tal naturaleza.

Sumado a lo anterior, se evidencia un dato aún más preocupante: en las 39 IES que ofrecen 42 cursos relacionados con la IHC -exceptuando categoría Proyectos-, se encontró que aun cuando 30 de ellos son obligatorios, sólo 9 corresponden a la categoría $\mathrm{IHC}$, incluso cuando el total de la categoría asciende a 14. Es decir, los cursos en los que se considera se otorga una visión integral y una importancia mayor a tal disciplina de forma Obligatoria, solo sumó el $6.57 \%$. En consecuencia, podría afirmarse que si la cobertura de los currículos sobre cursos relacionados con la IHC, se midiera por su cantidad, tal indicador apenas superaría el $6 \%$ y, por tanto, es 
considerablemente bajo. La figura 6 presenta la diferenciación de las asignaturas clasificadas en la ca- tegoría IHC, una vez se consolidaron los resultados de la fase de Revisión y Consulta.

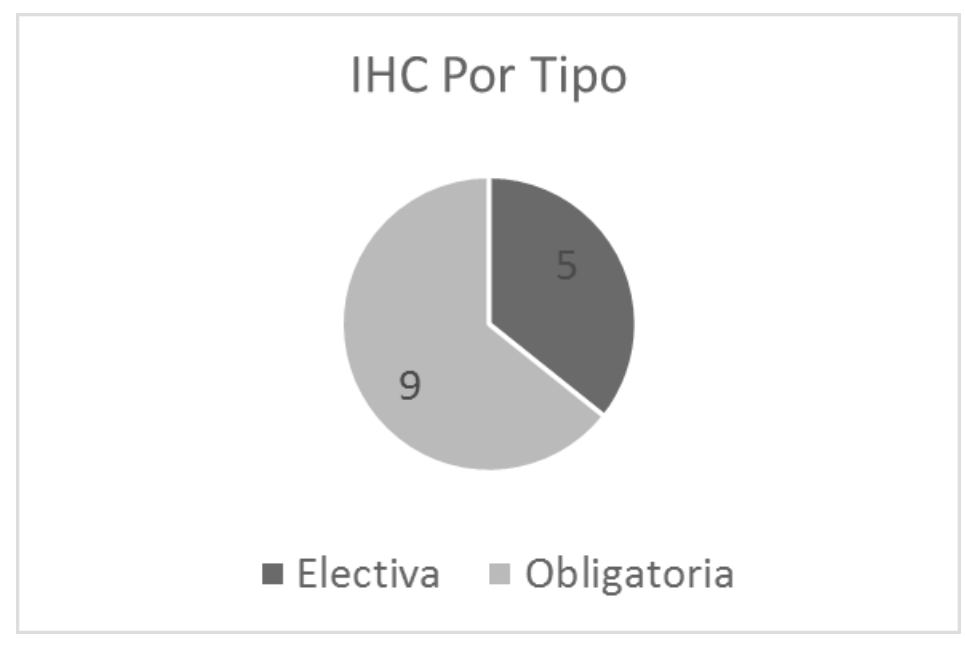

Figura 6. Asignaturas IHC por tipo.

En síntesis, podría considerarse que la baja cobertura de los currículos en asignaturas relacionadas con la IHC, puede generar una brecha en términos de habilidades en el sector productivo de la industria colombiana de software; a su vez, las organizaciones ubicadas en dicho sector tendrían que aumentar los costos de capacitación y entrenamiento en áreas de conocimiento relacionadas con la IHC, si lo que desean es disminuir esta brecha.
Adicionalmente, en el año 2015 en Colombia se graduaron 6.861 profesionales en Ingeniería de Sistemas, Telemáticas y afines (SNIES, 2017), el mismo núcleo básico usado para determinar la totalidad de las IES con programas objeto de estudio en la presente investigación. Las IES que incluyen en sus pénsum asignaturas relacionadas con la IHC de manera obligatoria se listan en la tabla 4, junto con la cantidad de graduados por cada institución para el año 2015.

\begin{tabular}{ll}
\hline IES & Graduados 2015 \\
\hline Pontificia Universidad Javeriana & 49 \\
Universidad Autónoma de Bucaramanga & 10 \\
Universidad Autónoma de Occidente & 34 \\
Universidad de Cartagena & 41 \\
Universidad de la Sabana & 19 \\
Universidad de Santander & 14 \\
Universidad Mariana & 35 \\
Universidad Santo Tomás & 41 \\
Universidad Manuela Beltrán & 56 \\
Total graduados de IES con asignaturas IHC obligatorias & 299 \\
Total graduados de todas las IES & 6861 \\
\hline
\end{tabular}

Tabla 4. Número de graduados de IES con asignaturas IHC obligatorias. 
La tabla 4 permite calcular que, al menos el $4.36 \%$ de los graduados del 2015, en carreras profesionales del núcleo básico objeto de estudio, obtuvieron una formación que incluye a la IHC como disciplina; en otras palabras, el sector productivo recibió para tal año a 6.562 profesionales no necesariamente capacitados en el diseño de sistemas interactivos con componentes de usabilidad, accesibilidad y todos aquellos que son estudiados en asignaturas relacionadas con la IHC.

Los datos aquí presentados son consistentes con los de otros investigadores, como los encontrados en Collazos y Merchan (2015) e invitan a las IES a replantear sus currículos con el fin de incluir en ellos asignaturas obligatorias en el campo de la IHC, con el ánimo de contribuir gradualmente con la formación de profesionales capaces de considerar dicha disciplina y lo que implica en la labor de desarrollar software.

Finalmente, el estudio cuantitativo que se presentó tiene limitaciones en relación con el alcance de las posibles causas que generan que las IES no incluyan en sus currículos asignaturas inherentes a la IHC. Dichas causas pueden ir desde cumplimiento de líneas de especialización establecidas por cada institución, la falta de personal docente especializado en tal campo o en el peor de los casos por la desatención y desinterés de las IES por dicha disciplina. En consecuencia, es prudente considerar estudios de naturaleza cualitativa que evalúen con mayor nivel de detalle lo que claramente se traduce como un cuestionamiento pendiente por resolver.

\section{Conclusiones}

El estudio comprendió las IES del sector público y privado en Colombia a nivel de pregrado. En este sentido, podría plantearse que un estudio que considere IES con programas a nivel de posgrado podría vislumbrar si áreas de conocimiento asociadas a la IHC han migrado a un nivel de educación más especializado y por ende de menor alcance para un mayor número de estudiantes.
Aunque los resultados muestran que solamente 9 cursos de la categoría IHC son de carácter obligatorio, se destaca que otros 20 cursos -exceptuando la categoría Proyectos- con la misma característica hacen parte de alguna de las áreas relacionadas. Esto conduce a concluir que a pesar de que la IHC no se aborda mayoritariamente de manera integral, las IES sí están trabajando en disciplinas complementarias que, sin lugar a dudas, contribuyen con la formación de profesionales con habilidades en el ámbito de la interacción, usabilidad y accesibilidad, entre otros.

Las necesidades del mercado, relacionadas con la producción de software se encuentran en constante cambio, por lo tanto, es necesario replantear la manera en que las IES forman a sus estudiantes, para que una vez egresados, también demuestren conocimientos relacionados con la interacción humano computador, con el fin de posibilitar su aplicación en la actividad laboral y, por tanto, contribuir con la calidad de los sistemas interactivos que serán desarrollados. En consecuencia, podría considerarse que los Ministerios de Educación Nacional (MEN) y el de las Tecnologías de la Información y las Comunicaciones (MINTIC) en común acuerdo fomenten iniciativas en pro de la formación de profesionales en el campo de la IHC.

Finalmente, este estudio no buscó la comprobación o negación de hipótesis en relación con los efectos de la oferta por parte de las IES, de cursos relacionados con la IHC en la industria colombiana de software; sin embargo, es un referente para estudios de la misma naturaleza, ya que provee desde una dimensión cuantitativa un análisis que podría abordar un estudio de mayor profundidad, con el fin de asegurar de manera precisa una posible relación causa-efecto, desde las perspectivas de los diversos actores que se encuentran alrededor del desarrollo de software.

\section{Agradecimientos}

Esta investigación no habría sido posible sin la valiosa ayuda de las IES que contribuyeron respon- 
diendo al cuestionario enviado a sus correos institucionales, a ellas muchas gracias. Igualmente, gracias a quienes enriquecieron este artículo con sus conceptos, críticas y opiniones; en especial a la Ph.D. María Patricia Trujillo Uribe, directora del grupo de investigación Multimedia y Visión por Computador, así como al Ing. Mauricio Eduardo Fernández Narváez, director del programa de Tecnología en Sistemas de Información, ambos de la Universidad del Valle.

\section{Referencias}

Abreu, J. (2012). Hipótesis, Método \& Diseño de Investigación. Daena: International Journal of Good Conscience, 7 (2), 187-197.

ACM., \& IEEE. (2017). Information Technology Curricula 2017: Curriculum Guidelines for Baccalaureate Degree Programs in Information Technology. doi: 10.1145/3173161

Azcona, M., Dorati, J., \& Manzini, F. (2013). Precisiones metodológicas sobre la unidad de análisis y la unidad de observación. IV Congreso Internacional de Investigación de la Facultad de Psicología de la Universidad Nacional de La Plata. La Plata, Argentina.

Cazau, P. (2006). Introducción a la Investigación en Ciencias Sociales. Buenos Aires, Argentina.

Collazos, C. A., Ortega, M., Granollers, T., Rusu, C., \& Gutierrez, F. L. (2016). Human-Computer Interaction in Ibero-America: Academic, Research, and Professional Issues. IT Professional, 18 (2), 8-11. doi: 10.1109/MITP.2016.38

Collazos, C. A., \& Merchán, L. (2015). Human-computer interaction in Colombia: bridging the gap between education and industry. IT Professional, 17 (1), 5-9. doi: 10.1109/MITP.2015.8

Ferreras, B., \& Hayser, J. (2008). Aplicación de la usabilidad al proceso de desarrollo de páginas web
(Tesis Doctoral). Recuperado de: http://oa.upm. es/1176/

Granollers, T. (2004). MPIU+a: Una metodología que integra la ingeniería del software, la interacción persona-ordenador y la accesibilidad en el contexto de equipos de desarrollo multidisciplinares. Lleida, España: Universidad de Lleida.

Granollers, T., Collazos, C. A., \& González, M. P. (2008). The State of $\mathrm{HCl}$ in Ibero-American countries. Journal of Universal Computer Science, 14 (6), 2599-2613. doi: JUCS-014-16-2599

Granollers, T., Lorés. J., \& Cañas. J. (2005). Diseño de sistemas interactivos centrados en el usuario. Barcelona, España: Editorial UOC.

ISO 25000. (2011). ISO/IEC 25010. Recuperado de: http://iso25000.com/index.php/normas-iso-25000/iso-25010

Marco-Simó, J. M, Marco-Galindo, M. J., \& Terrén, D. (2015). Casi le damos la vuelta a la enseñanza del desarrollo de software. XXI Jornadas sobre la Enseñanza Universitaria de la Informática, 341-349.

Marco-Simó, J. M, Guitart, I., \& Marco-Galindo, M. J. (2008). ¿Podemos darle la vuelta a la enseñanza del desarrollo del software?. Novatica: revista de la Asociación de Técnicos de Informática. 193, 59-62.

Mercado-Ramos, V. H., Zapata, J., \& Ceballos, Y. F. (2015). Herramientas y buenas prácticas para el aseguramiento de calidad de software con metodologías ágiles. Revista de Investigación, Desarrollo e Innovación, 6 (1), 73-83. doi: https://doi. org/10.19053/20278306.3277

Pressman, R. (2010). Ingeniería del Software. Un enfoque práctico. Ciudad de México, México: McGraw Hill.

SNIES. (2017). Observatorio laboral para la educación. Recuperado de: http://www.mineducacion. 
gov.co/sistemasdeinformacion/1735/w3-article-212301.html

Villegas, M., Hernández, H., \& Giraldo, W. (2009). Implementing a collaborative virtual environment - specification for a usability metamodel. Ingeniería e Investigación, 29 (1), 126-132. Recuperado de: https://revistas.unal.edu.co/index.php/ingeinv/ article/view/15152/34426

Villegas, M. L., Giraldo, W. J., Granollers, T., \& Collazos, C. A. (2007). Una propuesta de inclusión del área de Usabilidad en la estructura curricular en Colombia. Congreso Internacional de Interacción Persona-Ordenador, 193-202. Zaragoza, España. 